Canadian

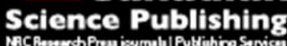

Applied Physiology, Nutrition, and Metabolism Physiologie appliquée, nutrition et métabolisme

\title{
Wholegrain pasta reduces appetite and meal-induced thermogenesis acutely: A pilot study
}

\begin{tabular}{|r|l|}
\hline Journal: & Applied Physiology, Nutrition, and Metabolism \\
\hline Manuscript ID & apnm-2015-0446.R2 \\
\hline Manuscript Type: & Article \\
\hline Date Submitted by the Author: & 10 -Nov-2015 \\
\hline & $\begin{array}{l}\text { Complete List of Authors: } \\
\text { Cacone, Roberto; Federico II University } \\
\text { Santarpia, Lidia; Federico II University } \\
\text { Iacro, Maurizio; Federico II University } \\
\text { Contaldo, Franco; Federico II University } \\
\text { Kristensen, Mette; Univ Copenhagen } \\
\text { Pasanisi, Fabrizio; Federico II University }\end{array}$ \\
\hline Keyword: & $\begin{array}{l}\text { Whole grain pasta, Fullness, Legumes, Meal - induced Thermogenesis, } \\
\text { Glucose metabolism }\end{array}$ \\
\hline \multicolumn{2}{|c}{} \\
\hline
\end{tabular}




\section{Wholegrain pasta reduces appetite and meal-induced thermogenesis acutely: A pilot study}

Iolanda Cioffi ${ }^{a}$, Lidia Santarpia $^{a}$, Andrea Vaccaro $^{a}$, Roberto Iacone $^{a}$, Giuseppe Labruna $^{b}$, $^{-}$ Maurizio Marra $^{a}$, Franco Contaldo $^{a c}{ }^{\text {, Mette Kristensen }}{ }^{d}$, Fabrizio Pasanisi $^{a c}$

${ }^{a}$ Internal Medicine and Clinical Nutrition Unit, Federico II University Hospital, via Pansini 5, 80131 Naples, Italy. ${ }^{b}$ IRCCS SDN, via E Gianturco 113, 80143 Naples and CEINGE-Biotecnologie Avanzate, via G. Salvatore 486, 80145 Naples, Italy. ${ }^{c}$ Interuniversity Center for Obesity and Eating disorders (CISRO), Department of Clinical Medicine and Surgery, Federico II University Hospital, via Pansini 5, 80131 Naples, Italy. ${ }^{d}$ Department of Nutrition, Exercise and Sports, Faculty of Science, University of Copenhagen, Rolighedsvej 30, DK-1958 Frederiksberg C.

\section{Corresponding author:}

Iolanda Cioffi, PhD

Department of Clinical Medicine and Surgery

Federico II University Hospital

Via Pansini 5

80131 Naples, Italy

iolanda.cioffi@unina.it; Tel.: +390817462333 fax: 0815466152. 


\section{Abstract}

The intake of foods rich in dietary fiber is associated with reduced risk to develop overweight and type 2 diabetes (T2D) in epidemiological studies. The present work aims to identify acute strategies to regulate appetite and improve glucose control by using different pasta meals. Hence, four different iso-caloric lunch meals were administered to eight healthy participants, in a crossover design, consisting of 1) refined grain pasta 2) whole-grain pasta 3) lemon juice-supplemented refined grain pasta and 4) refined grain pasta with legumes $(\mathrm{RG}+\mathrm{T}$; $\mathrm{WG}+\mathrm{T}$; $\mathrm{LRG}+\mathrm{T}$ and $\mathrm{RG}+\mathrm{L}$, respectively). On the test days, participants underwent baseline measurements, including appetite sensation, blood sample and resting energy expenditure (REE), hereafter, the test lunch was served. Subjective appetite was assessed and blood sample was taken each hour for 240 minutes while postprandial EE was measured for 3 hours. In repeated measures ANCOVA, postprandial fullness $(p=0.001)$ increased and hunger $(p=0.038)$ decreased. WG+T had a lower EE than both LGR+T $(p=0.02)$ and $\mathrm{RG}+\mathrm{L}(p<0.001)$. Likewise, meal-induced thermogenesis (MIT) was lower for $\mathrm{WG}+\mathrm{T}$ compared to $\mathrm{RG}+\mathrm{L}(58 \pm 81 \mathrm{~kJ}$ vs $248 \pm 188 \mathrm{~kJ} ; p<0.05)$. Plasma glucose $(p=0.001)$ was lower for $\mathrm{RG}+\mathrm{T}$ and triacylglycerols $(p=0.02)$ increased for $\mathrm{LRG}+\mathrm{T}$, however insulin, c-peptide and ghrelin did not differ compared to other meals. In conclusion, our study indicate that acute consumption of WG pasta may promote fullness and reduce hunger, lowering postprandial thermogenesis; whereas adding lemon juice to the pasta or legumes does not appear to affect appetite. However, none of pasta meal alterations improved postprandial metabolic profile.

\section{Keywords}

Whole grain pasta, Fullness, Legumes, Meal - induced Thermogenesis, Glucose metabolism. 


\section{Introduction}

A fiber-enriched and low glycemic index (GI) diet is a promising strategy against the increasing epidemic of overweight, obesity and non-communicable chronic diseases (NCCD) such as metabolic syndrome, T2D and cardiovascular diseases (CVD) (Feldeisen and Tucker 2007; Ye EQ et al. 2012). Results from prospective cohort studies have reported an inverse relationship between whole grain (WG) food consumption and body mass index (BMI) and/or abdominal fat (Harris and Kris-Etherton 2012; Liu 2002), although intervention trials conducted to date have failed to demonstrate beneficial effects on weight loss (Jonnalagadda et al. 2011). WG consumption may play a role in body weight regulation, potentially improving metabolic abnormalities associated with the development of T2D and CVD (Harris and Kris-Etherton 2012; Liu 2002). Thus, they have been observed to lower the glycemic response (Jenkins et al. 2012; Liu 2002) mainly due to the structure of WG and their particle size (Gross et al. 2004) that influence the rate of digestion and the consequent metabolic properties (Priebe et al. 2008). Further, WG foods are less energy dense than their refined counterparts (Harris and Kris-Etherton 2012) due to high dietary fiber content which may reduce the short-term appetite (Wanders et al. 2011) and affect EE (Khossousi et al. 2008). Although previous studies (Raben et al. 1994) observed decreased postprandial thermogenesis and increased fullness after a high-fiber meal, the impact of fiber-rich diet or meal on energy metabolism has not been well understood.

Likewise, legumes consumption could lead to weight loss (Abete et al. 2009; Crujeiras et al. 2007; Jenkins et al. 2012;) and their intake resulted in improving glycemic control (Sievenpiper et al. 2009). Not only are legumes good sources of slowly digested starch, but they are also relatively high in fiber and low in energy density (McCrory et al. 2010) and, hence, their regular consumptions may increase acute satiety (Li et al. 2014). However, legumes are commonly consumed with high-carbohydrate foods such as pasta, rice or bread and how their incorporation 
within a high carbohydrate meal could influence plasma glucose and subjective appetite is still poorly understood (Mollard et al. 2012).

Finally, some studies reported that bread products with added organic acids could be useful in improving glucose tolerance to starch and in lowering postprandial glucose and insulin responses (Liljeberg and Björck 1996). Improved glucose tolerance may result from the presence of organic acids that may delay gastric emptying or inhibit amylase activity and, hence, reduce the rate of carbohydrate absorption in the small intestine (Priebe et al. 2008). Therefore, in the present pilot study, different strategies to suppress subjective appetite and improve glycemic control have been applied. We tested the hypothesis that the following alterations of a traditional pasta meal: 1) Replacing refined pasta for WG pasta, and 2) Adding acid in the form of lemon juice to refined pasta would affect appetite and glycemic response, respectively. Then, we hypothesized that incorporating legumes into a traditional pasta meal would be an additional strategy to induce satiety and influence glucose metabolism. In addition, postprandial thermogenesis, triglycerides and ghrelin response were assessed.

\section{Materials and Methods}

\section{Study population}

Eight healthy volunteers ( 4 females and 4 males) were recruited for this pilot study. Inclusion criteria were BMI of 20-27 kg/m² and age 25-65 years. Exclusion criteria were: known chronic illnesses, diabetes, hypertension, hyperlipidemia, smoking, athletic physical activity, daily use of prescription medication (except for oral contraceptives and hormone replacement therapy (HRT)), 
use of dietary supplements, allergies or food intolerances or dislikes of relevance to the composition of meals.

\section{Study design}

Four different iso-caloric lunch meals were tested in 8 participants who all underwent one test day per week. The day before the test days the subjects were asked to follow a standardized fasting procedure, i.e. abstention from alcohol and hard physical activity and fasting from 10 p.m.. On the test days, participants were not allowed to smoke and they were instructed to consume a standardized breakfast meal at home (8.00 a.m.) within 10-12 min, which consisted of $200 \mathrm{ml}$ of semi-skimmed milk and $25 \mathrm{~g}$ of cornflakes (766 kJ; 18\% protein, $65 \%$ carbohydrate and $16 \%$ fat). Then, they reached the Department of Clinical Medicine and Surgery, Internal Medicine and Clinical Nutrition Unit, at Federico II University Hospital, in Naples (Italy) at 11.00 a.m. by calm mode of transportation. At their arrival, participants were weighed to the nearest $0.1 \mathrm{~kg}$ with a platform beam scale and height was measured to the nearest $0.5 \mathrm{~cm}$ using a wall-mounted stadiometer (only at the first visit). Firstly, baseline EE was measured by indirect calorimetry then, blood samples were taken and the subjects' appetite sensation was assessed using a Visual Analogue Scale (VAS) questionnaire (time point $0 \mathrm{~min}$ ). Then the test meal was served and consumed within 15 minutes. Palatability was assessed using VAS after the meal test. Blood samples and appetite sensation were measured every hour for a 4-hour period (time points 60, 120, 180 and $240 \mathrm{~min}$ ). Postprandial EE was measured for a period of 3 hours, counted from the beginning of test meal, with breaks of 10 minutes each hour for blood sampling and appetite ratings. The study was conducted in according to the Declaration of Helsinki and received the approval of the Local Ethical Committee. All participants provided written informed consent prior to enrolment. 


\section{Experimental meals}

Food used for the study was provided by the Italian pasta company "La Fabbrica della Pasta di Gragnano" (La Fabbrica della Pasta di Gragnano s.r.1., Gragnano, Italy). Pasta used for the current project is classified as PGI (Protected Geographical Indication) according to the European Council Regulation (EC) No 510/2006.

PGI requirements are as follow: pasta must be produced within Gragnano's city only with durum wheat semolina and water from local aquifers. The dough extrusion has to be done through bronze dies and pasta drying must be accomplished at a temperature between $40^{\circ}$ and $80^{\circ} \mathrm{C}$ and for 6 up to 60 hours. (www.politicheagricole.it/flex/cm/pages/ServeBLOB.php/L/IT/IDPagina/3348). Tomato and legume based sauces were prepared by "La Fabbrica della Pasta di Gragnano", frozen and then delivered at kitchen laboratory in Naples. Tomato sauce recipe for 5 servings was as follow: $700 \mathrm{ml}$ tomato puree, $30 \mathrm{~g}$ extra-virgin olive oil, $8 \mathrm{~g}$ garlic, $2 \mathrm{~g}$ fresh basil, $4 \mathrm{~g}$ salt and it was cooked for about 60 min. As legumes, we used brown beans Roviotti, prepared in a single batch as follow: $500 \mathrm{~g}$ dry beans, $60 \mathrm{~g}$ extra-virgin olive oil, $8 \mathrm{~g}$ garlic, $4 \mathrm{~g}$ salt and cooked for about 2 hours.

The test meals were prepared at the kitchen laboratory of the Department of Clinical Medicine and Surgery, Federico II University. They consisted of four pasta meals as follow: 1) $100 \mathrm{~g}$ refined grain pasta with $100 \mathrm{~g}$ tomato sauce $(\mathrm{RG}+\mathrm{T}) ; 2) 100 \mathrm{~g}$ WG pasta with $100 \mathrm{~g}$ tomato sauce $(\mathrm{WG}+\mathrm{T}) ; 3)$ $100 \mathrm{~g}$ lemon juice-supplemented refined grain pasta with $100 \mathrm{~g}$ tomato sauce (LRG+T); and 4) 70g refined grain pasta with $150 \mathrm{~g}$ legume based sauce $(\mathrm{RG}+\mathrm{L})$ (Table 1).

\section{Subjective appetite and palatability measurements}

VAS were used to assess subjective appetite sensation (hunger, satiety, fullness, prospective food consumption, thirst and well-being) and palatability (appearance, smell, taste, off taste and overall 
pleasantness) of the meal test. VAS are $100 \mathrm{~mm}$ in length with words anchored at each end, expressing the most positive and the most negative rating (Flint et al. 2000).

\section{Meal-Induced Thermogenesis (MIT) measurement}

MIT is the energy required for the processing and digestion of food consumed. It was calculated as the increase in EE over $3 \mathrm{~h}$ after lunch tests above the pre-meal EE used as baseline measure and divided by the energy content of each meal administered. MIT was expressed as both absolute value and percentage of the total energy of the test meal (Ruddick-Collins et al. 2013).

Pre- and post-prandial EE were measured by indirect calorimetry (Vmax29, Sensor Medics, Anaheim, California) with a ventilated hood and canopy system. The instrument was checked by burning ethanol, and oxygen and carbon dioxide analyzers were calibrated using nitrogen and standardized gases (mixtures of nitrogen, carbon dioxide and oxygen) before every run. Preprandial EE measurement was performed about 4 hours after breakfast $(766 \mathrm{~kJ})$ to avoid the thermic effect of previous meal (Compher et al. 2006). Participants were asked to lie down on a bed for at least $10 \mathrm{~min}$ in a quiet environment and at room temperature of $23-25^{\circ} \mathrm{C}$ (Marra et al. 2007). Then, oxygen and carbon dioxide production were determined for $30 \mathrm{~min}$, where the first 10 minutes were discarded from the analysis, to ensure that it was measured during a stable period. This measurement served as the "baseline" energy expenditure for the calculation of MIT. Immediately after meal test consumption, post-prandial EE was measured for 3 hours with two 10 min breaks at 60 and 120 minutes, respectively. Hence, the first 5 min of each measurement were excluded from the calculations in order to minimize the movement effects. EE was calculated with the abbreviated Weir's formula, neglecting protein oxidation (Weirs 1949).

Blood samples analysis 
Blood samples were drawn before the test meal and post-prandially at different time points (60, 120,180 and 240 minutes). Blood specimens were immediately centrifuged and stored at $-80^{\circ} \mathrm{C}$ until the analysis. TAG and plasma glucose concentrations were measured by automated methods (ABX Pentra 400, HORIBA ABX - Rome, Italy). The assays of c-peptide and insulin serum concentrations were performed by automatic ELISA Triturus analyzer (Biomedical Diagnostics Antwerpen, Belgium) and ELISA kits (Diasource Immunoassays S.A. - Louvain la Neuve, Belgium). For glucose, TAG, insulin and c-peptide the coefficient of variation (CV) were $0.6 \%, 1.7$ $\%, 5.1 \%$ and $4.8 \%$, respectively. Finally, ghrelin was assayed by Bio-Plex suspension array system (Bio-Rad Laboratories, CA, USA). The intra-assay and inter-assay CV were $2.5 \%$ and $0.9 \%$ respectively.

\section{Calculations and statistical analysis}

The incremental area under the curve (iAUC) was calculated as the net increment/decrement area above/below baseline value and AUC as the total area under the curve using the trapezoid model. All statistical analyses were performed using the Statistical Analysis System software package, version 9.4 (SAS Institute Inc., Cary, NC, USA). All dependent variables were controlled for homogeneity of variance and normal distribution by investigation of residual plots and normal probability plots and histograms, respectively. If the distribution of a variable was skewed, it was log-transformed prior to analyses and back-transformed before presentation. A repeated-measures ANCOVA analysis was used to examine the effect of meal and time and the meal $\times$ time interaction term on the postprandial response in PROC MIXED, where subject was modeled as a random variable and corresponding baseline value and BMI were modeled as covariates, and sex was included as fixed variable. An ANCOVA was used to examine the effect of meal on palatability and iAUC/AUCs in PROC MIXED, where subject was modeled as a random variable and the baseline 
values and BMI were modeled as covariates, and sex included as a fixed variable. The meal $\times$ time interaction term was removed when $\mathrm{p}>0.10$. Unadjusted post hoc pairwise comparisons were made for meal or for meal $\times$ time, when $\mathrm{p}>0.10$. All data are presented as means \pm standard deviations (SD) unless otherwise stated and the statistical significance level is defined as $\mathrm{p}<0.05$, whereas tendencies are considered to be present when $\mathrm{p}<0.10$.

\section{Results}

All subjects completed the RG+T test meal, 7 completed the $\mathrm{LRG}+\mathrm{T}$ and $\mathrm{WG}+\mathrm{T}$ meals and only 6 subjects completed the RG+L meal. Participants had a mean age of $39 \pm 14$ years, weight of $70.8 \pm 11.3 \mathrm{~kg}$ and a mean BMI of $24.7 \pm 2.7 \mathrm{~kg} / \mathrm{m}^{2}$. Two participants dropped out due to personal reasons unrelated to the study.

Subjective appetite sensation and palatability of the test meals

Fullness, satiety and prospective food intake ratings did not differ between meals at baseline. Hunger ratings differed between meals at baseline $(\mathrm{p}<0.05)$ with the lowest hunger ratings for $\mathrm{RG}+\mathrm{T}(44 \pm 20 \mathrm{~mm})$ compared to both $\mathrm{LRG}+\mathrm{T}(67 \pm 11 \mathrm{~mm})$ and $\mathrm{WG}+\mathrm{T}(67 \pm 9 \mathrm{~mm})(\mathrm{p}<0.05)$, but not $\mathrm{RG}+\mathrm{L}(57 \pm 21 \mathrm{~mm})$. In the repeated measures ANCOVA, postprandial satiety ratings did not differ between meals $(p=0.30)$, whereas ratings of fullness $(p=0.001)$, hunger $(p=0.038)$ and prospective food intake $(\mathrm{p}=0.034)$ did. Generally, $\mathrm{WG}+\mathrm{T}$ resulted in significantly greater fullness and reduced hunger and prospective food intake ratings compared to all other meals $(\mathrm{p}<0.02)$ (Figure 1), whereas no differences emerged between $R G+T, L R G+T$ and $R G+L$. The AUC for satiety, hunger, fullness and prospective consumption did not differ between meals. Palatability 
ratings did not differ in term of appearance, smell, taste, off taste and overall pleasantness ( $\mathrm{p}>0.70)$ among the four different meals (Table 2).

\section{Energy expenditure and MIT}

Postprandial EE increased for all different meals as shown in Figure 2. Repeated measures ANCOVA showed that postprandial EE was affected both by meals $(p=0.004)$ and time $(p=0.002)$. Post hoc pairwise analysis revealed that $\mathrm{EE}$ for $\mathrm{WG}+\mathrm{T}$ was lower than $\mathrm{LGR}+\mathrm{T}(\mathrm{p}=0.02)$ and $\mathrm{RG}+\mathrm{L}$ $(p=0.0005)$, but not for $R G+T(p=0.21)$. $E E$ for $W G+T$ was lower than $E E$ for $R G+T$ at time point $60 \mathrm{~min}(\mathrm{p}=0.007)$ and was still reduced compared to $\mathrm{RG}+\mathrm{L}$ at time point $180 \mathrm{~min}(\mathrm{p}=0.002)$. The iAUC was significantly decreased for $\mathrm{WG}+\mathrm{T}$ compared to $\mathrm{RG}+\mathrm{L}(\mathrm{p}<0.02)$ and slightly lowered compared to $\mathrm{LRG}+\mathrm{T}(\mathrm{p}=0.06)$.

MIT was calculated as the post-prandial increase of EE over $3 \mathrm{~h}$ after meal (Table 3 ) and found lower for $\mathrm{WG}+\mathrm{T}$ compared to $\mathrm{RG}+\mathrm{L}(58 \pm 81 \mathrm{~kJ}$ vs $248 \pm 188 \mathrm{~kJ}$; $\mathrm{p}<0.05)$; also when expressed as percentage of the energy content of the test meal $(3.2 \%$ for $W G+T$ vs. $13.2 \%$ for $R G+L)(p<0.05)$.

Plasma glucose, insulin, C-peptide and TAG responses

Plasma glucose, insulin, c-peptide and TAG responses and their corresponding AUCs are shown in Figure 3. Glucose response was influenced by meals $(p<0.001)$. In details, an overall reduction in plasma glucose after $\mathrm{RG}+\mathrm{T}$ compared to $\mathrm{RG}+\mathrm{L}(\mathrm{p}<0.02), \mathrm{WG}+\mathrm{T}(\mathrm{p}<0.02)$ and $\mathrm{LRG}+\mathrm{L}(\mathrm{p}<0.001)$. On the other hand, the postprandial insulin response was slightly lower for both meals with the highest fiber content ( $\mathrm{WG}+\mathrm{T}$ and $\mathrm{RG}+\mathrm{L}$ ) than for the other meals $(\mathrm{RG}+\mathrm{T}$ and $\mathrm{LRG}+\mathrm{L})$, although it was not significant $(\mathrm{p}=0.60)$; while, $c$-peptide responses did not differ among meals $(\mathrm{p}=0.23)$. The AUCs for glucose, insulin and C-peptide were not different between meals $(p=0.30 ; p=0.40$; $\mathrm{p}=0.87$, respectively). Finally, plasma TAG concentration was significantly affected by different 
meals at baseline and consequently during postprandial period $(\mathrm{p}=0.02)$, however AUC was not different between meals $(\mathrm{p}=0.20)$.

\section{Ghrelin response}

Ghrelin response did not differ among meals at baseline. Neither the repeated measure ANCOVA nor analyses of the AUC for ghrelin revealed any differences in the ghrelin response to the different meals (data not shown).

\section{Discussion}

The hypothesis of the present study was that different alterations of the traditional pasta meal, i.e. substituting refined grains for whole grains, adding lemon juice to the pasta or incorporating legumes in the pasta sauce as further choice, would improve appetite and glucose response. We were however not able to substantiate our hypothesis entirely as our results showed that only $\mathrm{WG}+\mathrm{T}$ increased fullness without a concurrent reduction in postprandial glycaemia, but with a decreased postprandial EE; while, $\mathrm{LRG}+\mathrm{T}$ and $\mathrm{RG}+\mathrm{L}$ did not improve any of the measured outcomes when compared to RG+T.

WG food consumption has been proposed to play a role in affecting appetite (Jonnalagadda et al. 2011), although their effect depends on the type and the amount of dietary fiber (Wanders et al. 2011). Viscous fibers such as $\beta$-glucan, lupin kernel fiber, rye bran, whole grain rye, are thought to exert the greatest effects (Wanders et al. 2011); however non-viscous wheat fibers has been previously shown to form an homogeneous bolus in the stomach, not in the small intestine, compared to refined counterpart, likely due to the presence of arabinoxylans, delaying gastric 
emptying and potentially improving short-term appetite (Marciani et al. 2013). Thus, factors such as total fiber content and volume of the meal may be important to satiety, since they could physically prolong stomach distension, with an increased short-term satiety (Kristensen et al. 2010; Marciani et al. 2013). In addition, similar results on subjective appetite were found in a randomized crossover study performed in Denmark, where we again investigated the effect of WG pasta PGI in 16 participants on appetite and food intake (unpublished). But, a previous study focused on both pasta and bread conducted by Kristensen et al. (2010), showed that WG pasta compared to RG pasta did not influenced neither subjective appetite nor ad libitum energy intake, but as expected, pasta showed a lower glycaemic response compared to bread, due to low GI.

Then, pasta investigated in our works is a PGI pasta and likely, some properties such as semolina used for the dough and processing cycle, in particular drying temperature required for artisanal pasta production, could positively improve their sensory quality compared to industrial pasta (De Zorzi et al. 2007). Hence, to avoid any confounding factors related to the type of pasta PGI, we assessed palatability by VAS and we did not find any significant difference among meals as necessarily required for the study purpose (Sorensen et al. 2003).

As part of our hypothesis, glucose response would be influenced by pasta with lemon juice added in the dough due to the presence of acidic compounds within pasta since it has been shown to improve glucose tolerance (Liljeberg and Björck 1996). We found that LRG+T did not improve blood glucose and insulin response during post-prandial period, in contrast with previous findings on bread reported by Liljerberg et al. (1996), whilst the lowest plasma glucose response was observed for RG+T. However, it should be highlighted that pasta with lemon juice (LRG) was not specifically manufactured for the study aims therefore lemon concentration may not have been high enough to lower the glycemic and insulin response. Also $\mathrm{WG}+\mathrm{T}$ (total fiber content $=8.1 \mathrm{~g}$ ) did not affect glucose metabolism compared to refined counterpart $(\mathrm{RG}+\mathrm{T}=3.8 \mathrm{~g})$ as previously shown 
(Kristensen et al. 2010; Giacco et al. 2010), although not always seen (Liu 2002). Likely, the type of dietary fiber contained in WG pasta, mainly insoluble, primarily increase bulk rather than form viscous solutions upon hydration in the small intestine (Wanders et al. 2011), explaining this inconsistency.

Surprisingly, the legume meal $(\mathrm{RG}+\mathrm{L})$ did not enhanced neither appetite nor glucose response as hypothesized, despite its high fiber content and low GI (Jenkins et al. 2012). However, we did not evaluate only legume consumption that has been reported to be associated with increase of satiety (Li et al. 2014), but rather incorporation of legumes within a meal that also included refined pasta in order to reflect a typical meal. Likely, the combination of refined pasta with legumes could have influenced their physiological properties due to the presence of other components such as carbohydrates, fibers, fat, protein and other nutrients provided by co-ingested foods that might influence the composition and the availability of macronutrients (Bornet et al. 2007). Previously Mollard et al. (2011) showed that incorporating pulses into a high carbohydrate meal such as pasta did not result in affecting subjective appetite as we observed, although it can affect food intake within meal, which it was however dependent on pulse type.

Macronutrients composition was kept constant among RG+T, WG+T and $\mathrm{LRG}+\mathrm{T}$ meals, apart from dietary fibers, which were higher in the $\mathrm{WG}+\mathrm{T}$; while proteins were slightly higher in the $\mathrm{RG}+\mathrm{L}$ meal. This may explain why we found that $\mathrm{RG}+\mathrm{L}$ significantly increased $\mathrm{EE}$ in comparison with $\mathrm{WG}+\mathrm{T}$, which showed the lowest response. Our results showed that $\mathrm{WG}+\mathrm{T}$ decreased postprandial EE as previously shown (Raben et al. 1994; Scalfi et al. 1987), although the mechanism involved was not understood yet. According to Raben et al., (1994) neither fiber content nor palatability seemed to be responsible for that result, since we did not observe significant differences in both metabolic parameters and palatability among meals. On the other hand, we observed an increase of EE following $\mathrm{RG}+\mathrm{L}$ that was held higher from baseline until the end of 
measurement ( $3 \mathrm{~h}$ ), likely due to a slight higher protein content and energy density, which is responsible for higher thermogenesis. As reported by previous studies, thermogenesis derives for $20-30 \%$ from ingested proteins, 5-10\% from carbohydrates and 0-3\% from fat (vanBaak 2008). A longer observation time ( $>3 \mathrm{~h}$ ) should have been necessary to quantify the entire EE response of RG+L, however Ruddick-Collins at al. (2013), demonstrated that shorter measures strongly correlated with the $6 \mathrm{~h}$ postprandial EE measurement, therefore may provide sufficient information about their overall response.

Besides EE, we calculated MIT that has previously been suggested to be one of the mechanisms that influences appetite sensation, including satiety (Westerterp-Plantenga et al. 1999), even though data are still contrasting (Crovetti et al. 1998; Raben et al. 2003; Ravn et al. 2013; WesterterpPlantenga et al. 1999). In the present work, MIT increased following RG+L without influence on subjective appetite as shown in previous studies (Raben et al. 2003; Ravn et al. 2013). However, MIT was lower after WG+T with a concurrent increased of fullness sensation. Similarly, Raben et al. (1994) found that MIT and fullness inversely correlated with fiber content (4.7 g/MJ vs 1.7 $\mathrm{g} / \mathrm{MJ})$ of different iso-energetic meals. However, MIT reduction was not reflected in delaying postprandial glucose, insulin, c-peptide and TAG concentrations. Therefore, it is unlikely that MIT was altered by the presence of the dietary fiber used in this study, but this effect could be ascribed mainly to the composition of meal (Raben et al., 2003). Finally, we assessed ghrelin response, which resulted to be not affected by different meals $(\mathrm{RG}+\mathrm{T}, \mathrm{WG}+\mathrm{T}, \mathrm{LRG}+\mathrm{T}$ and $\mathrm{RG}+\mathrm{L})$ as already shown (Giacco et al. 2010; Hartvigsen et al. 2014).

Several factors in study design, other than the type of fiber, should be carefully considered when the effects of dietary fiber on appetite were evaluated such as the strategy of fiber supplementation (isolated fiber processed or naturally contained) or the study design itself (Wanders et al. 2011). As consequence, the limited sample size and some drops out in addition with some limitations within 
the study protocol such as lack of tools for breakfast compliance evaluation and late sampling points after meal tests could have prevented from appreciating acute effects of high fiber meal on post-prandial metabolic profile. In addition, meals were not matched for carbohydrate content and as shown in Table 1, there are some small differences among test meals, which may be regarded as further study limitation.

However, the number of subjects needed for studies assessing appetite can be reduced considerably by using paired designs, therefore it is possible to detect a difference in appetite scores with $\alpha=0.05$ and power of $80 \%$ using 8 subjects over a 4.5 hour test meal as shown by Flint et al. (2000). Presumably, if we had measured the ad libitum food intake at the subsequent meal we would have been useful to strengthen the overall WG effect on appetite.

In conclusion, this pilot study shows that WG pasta PGI may promote acute fullness, and is well accepted among participants according to the palatability test. Although we observed an interesting reduction in postprandial thermogenesis, $\mathrm{WG}+\mathrm{T}$ did not influence the postprandial metabolic profile. On the other hand, the incorporation of pulses into a high carbohydrate meal did not show any significant effect on appetite and glucose response at least in the short-term. However, much research is required to better understand any possible WG effect on appetite regulation in a larger population. 


\section{Acknowledgements}

This study was supported by POR CAMPANIA FSE 2007/2013, Project CARINA. The authors gratefully acknowledge Marianna Naccarato for her assistance in the supervision to the preparation of meals and all participants enrolled in the study. The authors declare no conflict of interest. 


\section{References}

Abete, I., Parra, D., Martinez, J.A. 2009. Legume-, fish-, or high-protein-based hypocaloric diets: effects on weight loss and mitochondrial oxidation in obese men. J Med Food, 12(1): 100-8. PMID: 19298202.

Bornet, F.R., Jardy-Gennetier, A.E., Jacquet, N., Stowell, J. 2007. Glycaemic response to foods: impact on satiety and long-term weight regulation. Appetite, 49(3): 535-53. PMID: 17610996.

Clark, M.J., Slavin, J.L. 2013.The effect of fiber on satiety and food intake: a systematic review. J Am Coll Nutr. 32(3): 200-11. PMID: 23885994.

Compher, C., Frankenfield, D., Keim, N., Roth-Yousey, L. 2006. Evidence Analysis Working Group. Best practice methods to apply to measurement of resting metabolic rate in adults: a systematic review. J Am Diet Assoc. 106(6): 881-903. PMID: 16720129.

Crovetti, R., Porrini, M., Santangelo, A., Testolin, G. 1998. The influence of thermic effect of food on satiety. Eur J Clin Nutr. 52(7): 482-8. PMID: $\underline{9683329}$.

Crujeiras, A.B., Parra, D., Abete, I., Martinez, J.A. 2007. A hypocaloric diet enriched in legumes specifically mitigates lipid peroxidation in obese subjects. Free Radic Res. 41(4): 498-506. PMID: 17454132.

De Zorzi, M., Curioni, A., Simonato, B., Giannattasio, M., Pasini, G. 2007. Effect of pasta drying temperature on gastrointestinal digestibility and allergenicity of durum wheat proteins, Food Chemistry, Volume 104, Issue 1, Pages 353-363. http://dx.doi.org/10.1016/j.foodchem.2006.11.057. Feldeisen, S.E., Tucker, K.L. 2007. Nutritional strategies in the prevention and treatment of metabolic syndrome. Appl Physiol Nutr Metab. 32(1):46-60. PMID: 17332784. 
Flint, A., Raben, A., Blundell, J. E., Astrup, A. 2000. Reproducibility, power and validity of visual analogue scales in assessment of appetite sensations in single test meal studies. International Journal of Obesity. 24(1): 38-48. PMID: 10702749.

Giacco, R., Clemente, G., Cipriano, D., Luongo, D., Viscovo, D., Patti, L., et al. 2010. Effects of the regular consumption of wholemeal wheat foods on cardiovascular risk factors in healthy people. Nutr Metab Cardiovasc Dis. 20(3): 186-94. PMID: 19502018.

Gross, L.S., Li, L., Ford, E.S., Liu, S. 2004. Increased consumption of refined carbohydrates and the epidemic of type 2 diabetes in the United States: an ecologic assessment. Am J Clin Nutr.79(5): 774-9. PMID: 15113714.

Harris, K.A., Kris-Etherton, P.M. 2010. Effects of whole grains on coronary heart disease risk. Curr Atheroscler Rep. 12(6): 368-76. PMID: 20820954.

Hartvigsen, M.L., Lærke, H.N., Overgaard, A., Holst, J.J., Bach Knudsen, K.E., Hermansen, K. 2014. Postprandial effects of test meals including concentrated arabinoxylan and whole grain rye in subjects with the metabolic syndrome: a randomised study. Eur J Clin Nutr. 68(5):567-74. doi: 10.1038/ejen.2014.25. PMID: 24595224.

Jenkins, D.A., Kendall, C.W.C., Augustin, L.S.A., Mitchell, S., Sahye-Pudaruth, S., Blanco Mejia, S., et al. 2012. Effect of legumes as part of a low glycemic index diet on glycemic control and cardiovascular risk factors in type 2 diabetes mellitus: a randomized controlled trial. Arch Intern Med;172(21): 1653-1660. PMID: 23089999.

Jonnalagadda, S.S., Harnack, L., Liu, R.H., McKeown, N., Seal, C., Liu, S., et al. 2011. Putting the whole grain puzzle together: health benefits associated with whole grains-summary of American Society for Nutrition 2010 Satellite Symposium. J Nutr. 141(5): 1011S-22S. PMID: 21451131. 
Khossousi, A., Binns, C.W., Dhaliwal, S.S., Pal, S. 2008. The acute effects of psyllium on postprandial lipaemia and thermogenesis in overweight and obese men. Br J Nutr. 99(5): 1068-75. PMID: 18005484.

Kristensen, M., Jensen, M.G., Riboldi, G., Petronio, M., Bügel, S., Toubro, S., et al. 2010. Wholegrain vs. refined wheat bread and pasta. Effect on postprandial glycemia, appetite, and subsequent ad libitum energy intake in young healthy adults. Appetite. 54(1): 163-9. PMID: 19837118.

Li, S.S., Kendall, C.W., de Souza, R.J., Jayalath, V.H., Cozma, A.I., Ha, V., et al. 2014. Dietary pulses, satiety and food intake: a systematic review and meta-analysis of acute feeding trials. Obesity (Silver Spring). 22(8): 1773-80. PMID: 24820437.

Liljeberg, H.G., Björck, I.M. 1996. Delayed gastric emptying rate as a potential mechanism for lowered glycemia after eating sourdough bread: studies in humans and rats using test products with added organic acids or an organic salt. Am JClin Nutr. 64(6): 886-93. PMID: $\underline{8942413 .}$

Liu, S. 2002. Intake of refined carbohydrates and whole grain foods in relation to risk of type 2 diabetes mellitus and coronary heart disease. J Am Coll Nutr. 21(4): 298-306. PMID: $\underline{12166526 .}$.

Marciani, L., Pritchard, S.E., Hellier-Woods, C., Costigan, C., Hoad, C.L., Gowland, P.A., et al. 2013. Delayed gastric emptying and reduced postprandial small bowel water content of equicaloric whole meal bread versus rice meals in healthy subjects: novel MRI insights. Eur J Clin Nutr. 67(7): 754-8. PMID: 23594839.

Marra, M., Pasanisi, F., Montagnese, C., De Filippo, E., De Caprio, C., de Magistris, L., et al. 2007. BMR variability in women of different weight. Clin Nutr.26:567-572. PMID: 17517450. 
McCrory, M.A., Hamaker, B.R., Lovejoy, J.C., Eichelsdoerfer, P.E. 2010. Pulse consumption, satiety, and weight management. Adv Nutr. 1(1): 17-30. PMID: 22043448. Mollard, R.C., Zykus, A., Luhovyy, B.L., Nunez, M.F., Wong, C.L., Anderson, G.H. 2012. The acute effects of a pulse-containing meal on glycaemic responses and measures of satiety and satiation within and at a later meal. Br J Nutr. 108(3): 509-17. PMID: 22054112. Priebe, M.G., van Binsbergen, J.J., de Vos, R., Vonk, R.J. 2008. Whole grain foods for the prevention of type 2 diabetes mellitus. Cochrane Database Syst Rev. 23(1):CD006061. PMID: 18254091.

Raben, A., Christensen, N.J., Madsen, J., Holst, J.J., Astrup, A. 1994. Decreased postprandial thermogenesis and fat oxidation but increased fullness after a high-fiber meal compared with a low-fiber meal. Am J Clin Nutr. 59(6): 1386-94. PMID: 8198065. Raben, A., Agerholm-Larsen, L., Flint, A., Holst, J.J., Astrup, A. 2003. Meals with similar energy densities but rich in protein, fat, carbohydrate, or alcohol have different effects on energy expenditure and substrate metabolism but not on appetite and energy intake. Am J Clin Nutr.77(1): 91-100. PMID: 12499328.

Ravn, A.M., Gregersen, N.T., Christensen, R., Rasmussen, L.G., Hels, O., Belza, et al. 2013. Thermic effect of a meal and appetite in adults: an individual participant data metaanalysis of meal-test trials. Food Nutr Res. 23;57. PMID: 24376394. Ruddick-Collins, L.C., King, N.A., Byrne, N.M., Wood, R.E. 2013. Methodological considerations for meal-induced thermogenesis: measurement duration and reproducibility. Br J Nutr. 14;110(11):1978-86. PMID: 23683799.

Scalfi, L., Coltorti, A., D’Arrigo, E., Carandente, V., Mazzacano, C., Di Palo, M., et al.1987. Effect of dietary fibre on postprandial thermogenesis. Int J Obes. 11, Suppl. 1,9599. PMID: 3032832. 
Sievenpiper, J.L., Kendall, C.W.C., Esfahani, A., Wong, J.M., Carleton, A.J., Jiang, H.Y., et al.2009. Effect of non-oil-seed pulses on glycaemic control: a systematic review and metaanalysis of randomised controlled experimental trials in people with and without diabetes. Diabetologia.52(8): 1479-1495. PMID: 19526214.

Sorensen, L., Moller, P., Flint, A., Martens, M., Raben, A. 2003. Effect of sensory perception of foods on appetite and food intake: a review of studies on humans. International Journal of Obesity. 27(10): 1152-1166. PMID: 14513063. vanBaak, M.A. 2008. Meal-induced activation of the sympathetic nervous system and its cardiovascular and thermogenic effects in man. Physiol Behav. 94(2): 178-86. PMID: $\underline{18281067 .}$.

Wanders, A.J., van den Borne, J.J., de Graaf, C., Hulshof, T., Jonathan, M.C., Kristensen M., et al. 2011. Effects of dietary fibre on subjective appetite, energy intake and body weight: a systematic review of randomized controlled trials. Obes Rev.12(9): 724-739. PMID: 21676152.

Weirs, J.B.D. 1949. New methods for calculating metabolic rate with specific reference to protein metabolism J Phisiol.109, pp. 1-9. PMID: 15394301.

Westerterp-Plantenga, M.S., Rolland, V., Wilson, S.A., Westerterp, K.R. 1999. Satiety related to $24 \mathrm{~h}$ diet-induced thermogenesis during high protein/carbohydrate vs. high fat diets measured in a respiration chamber. Eur J ClinNutr. 53(6):495-502. PMID: 10403587. Ye, E.Q., Chacko, S.A., Chou, E.L., Kugizaki, M., Liu, S. 2012. Greater whole-grain intake is associated with lower risk of type 2 diabetes, cardiovascular disease, and weight gain. $\mathrm{J}$ Nutr. 142(7):1304e13. doi: 10.3945/jn.111.155325. PMID: 22649266. 
Table 1. Macronutrient composition of the four tested meals.

\begin{tabular}{|c|c|c|c|c|}
\hline & RG+T & WG+T & LRG+T & $\mathbf{R G}+\mathbf{L}$ \\
\hline Meal weight $(\mathrm{g})$ & 333 & 312 & 316 & 312 \\
\hline Meal volume (ml) & 570 & 570 & 570 & 570 \\
\hline Uncooked pasta (g) & 100 & 100 & 100 & 70 \\
\hline Tomato sauce $(\mathrm{ml})$ & 100 & 100 & 100 & - \\
\hline Roviotti brown beans dry (g) & - & - & - & 50 \\
\hline Total Energy (kJ) & 1800 & 1766 & 1800 & 1884 \\
\hline Energy from carbohydrate (\%) & 75 & 72 & 75 & 69 \\
\hline Energy from fat $(\%)$ & 13 & 14 & 13 & 15 \\
\hline Energy from protein $(\%)$ & 12 & 14 & 12 & 16 \\
\hline Carbohydrate content (g) & 86.2 & 80.6 & 86.2 & 82.8 \\
\hline Fat content $(\mathrm{g})$ & 6.11 & 6.51 & 6.11 & 7.49 \\
\hline Protein content $(\mathrm{g})$ & 12.9 & 15.2 & 12.9 & 18.1 \\
\hline Fiber content $(\mathrm{g})$ & 3.8 & 8.1 & 3.4 & 10.5 \\
\hline
\end{tabular}

$\mathrm{RG}+\mathrm{T}=$ refined wheat pasta + Tomato sauce; $\mathrm{WG}+\mathrm{T}=$ whole grain wheat pasta + Tomato sauce; $\mathrm{LRG}+\mathrm{T}=$ lemon refined wheat pasta + Tomato sauce; $\mathrm{RG}+\mathrm{L}=$ refined wheat pasta + legumes. 
Table 2. Palatability ratings following the consumption of the four meals tested.

\begin{tabular}{lccccc}
\hline & Taste & Smell & Appearance & Off taste & Over-all pleasantness \\
\hline $\mathbf{R G}+\mathbf{T}$ & $17 \pm 13$ & $29 \pm 20$ & $33 \pm 26$ & $28 \pm 28$ & $23 \pm 17$ \\
$\mathbf{W G}+\mathbf{T}$ & $27 \pm 20$ & $18 \pm 18$ & $23 \pm 17$ & $24 \pm 24$ & $25 \pm 25$ \\
$\mathbf{L R G}+\mathbf{T}$ & $24 \pm 10$ & $23 \pm 13$ & $33 \pm 25$ & $20 \pm 12$ & $22 \pm 14$ \\
$\mathbf{R G}+\mathbf{L}$ & $20 \pm 18$ & $22 \pm 25$ & $30 \pm 19$ & $20 \pm 19$ & $23 \pm 19$ \\
\hline
\end{tabular}

Ratings expressed as mm (0-100). Data are presented as mean $\pm \mathrm{SD}$.

$\mathrm{RG}+\mathrm{T}=$ refined wheat pasta + Tomato sauce; $\mathrm{WG}+\mathrm{T}=$ whole grain wheat pasta + Tomato sauce;

$\mathrm{LRG}+\mathrm{T}=$ lemon refined wheat pasta + Tomato sauce; $\mathrm{RG}+\mathrm{L}=$ refined wheat pasta + legumes. $p=\mathrm{ns}$. 
Table 3. Meal-induced Thermogenesis (MIT) of the four meals.

\begin{tabular}{lcc}
\hline & MIT (kJ) & MIT (\%) \\
\hline RG+T & $193 \pm 197$ & $10.7 \pm 10.9$ \\
\hline WG+T & $58 \pm 81$ & $3.2 \pm 4.6$ \\
\hline LRG+T & $170 \pm 231$ & $9.4 \pm 12.8$ \\
\hline RG+L & $248 \pm 188$ & $13.2 \pm 9.9$ \\
\hline
\end{tabular}

MIT is reported in both absolute ( $\mathrm{kJ}$ above REE) values and percentage of energy of the test meal. Data are presented as mean $\pm \mathrm{SD} . \mathrm{RG}+\mathrm{T}=$ refined wheat pasta + Tomato sauce; $\mathrm{WG}+\mathrm{T}=$ whole grain wheat pasta + Tomato sauce; $\mathrm{LRG}+\mathrm{T}=$ lemon refined wheat pasta + Tomato sauce; $\mathrm{RG}+\mathrm{L}=$ refined wheat pasta + legumes. WG+T vs RG+L: $p<0.05$. 


\section{Figure legends}

\section{Figure 1}

Unadjusted mean ratings of satiety (a), fullness (b), hunger (c) and prospective food consumption (d) during $4 \mathrm{~h}$ following 4 lunch test meals $(\mathrm{RG}+\mathrm{T}=$ refined wheat pasta + Tomato sauce; $\mathrm{WG}+\mathrm{T}=$ whole grain wheat pasta + Tomato sauce; $\mathrm{LRG}+\mathrm{T}=$ lemon refined wheat pasta + Tomato sauce; $\mathrm{RG}+\mathrm{L}=$ refined wheat pasta + legumes) and the corresponding AUCs expressed as mean $\pm \mathrm{SEM}$ $(n=6)$.

\section{Figure 2}

Post-prandial energy expenditure (EE) are expressed as delta value $(\Delta)$ during $3 \mathrm{~h}$ following the 4 lunch test meals $(\mathrm{RG}+\mathrm{T}=$ refined wheat pasta + Tomato sauce; $\mathrm{WG}+\mathrm{T}=$ whole grain wheat pasta + Tomato sauce; $\mathrm{LRG}+\mathrm{T}=$ lemon refined wheat pasta + Tomato sauce; $\mathrm{RG}+\mathrm{L}=$ refined wheat pasta + legumes) and corresponding AUC and iAUC as mean $\pm \operatorname{SEM}(n=6) . *$ indicate a difference with $p<0.007$, \# indicate a difference with $p<0.002$ and different letters indicate a difference with $p=0.02$.

\section{Figure 3}

Unadjusted mean glucose (a), insulin (b), c-peptide (c) and TAG (d) concentrations during the $4 \mathrm{~h}$ after 4 lunch test meals $(\mathrm{RG}+\mathrm{T}=$ refined wheat pasta + Tomato sauce; $\mathrm{WG}+\mathrm{T}=$ whole grain wheat pasta + Tomato sauce; $\mathrm{LRG}+\mathrm{T}=$ lemon refined wheat pasta + Tomato sauce; $\mathrm{RG}+\mathrm{L}=$ refined wheat pasta + legumes) and corresponding AUCs expressed as mean $\pm \operatorname{SEM}(n=6)$. 

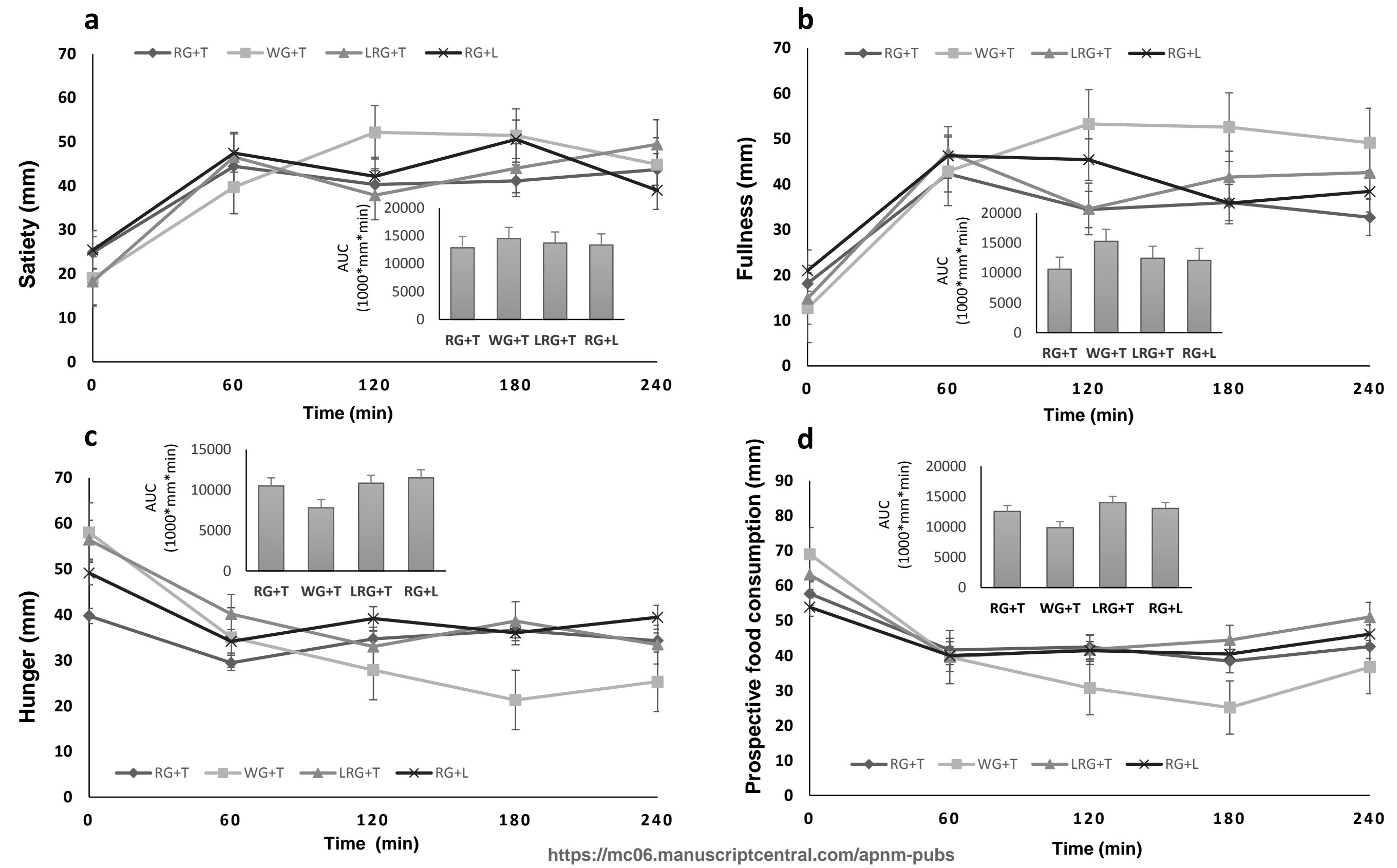

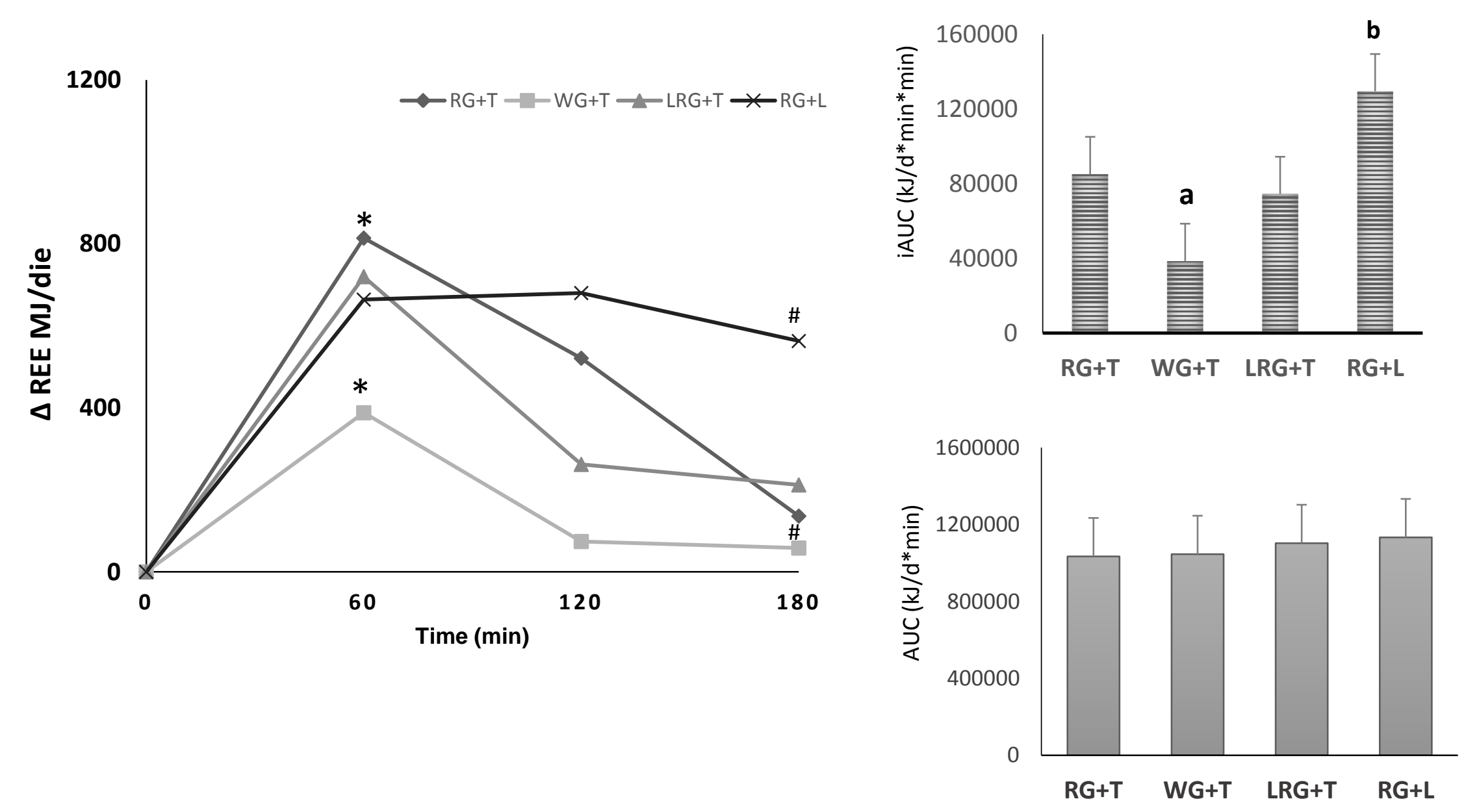

https://mc06.manuscriptcentral.com/apnm-pubs 


\section{a}
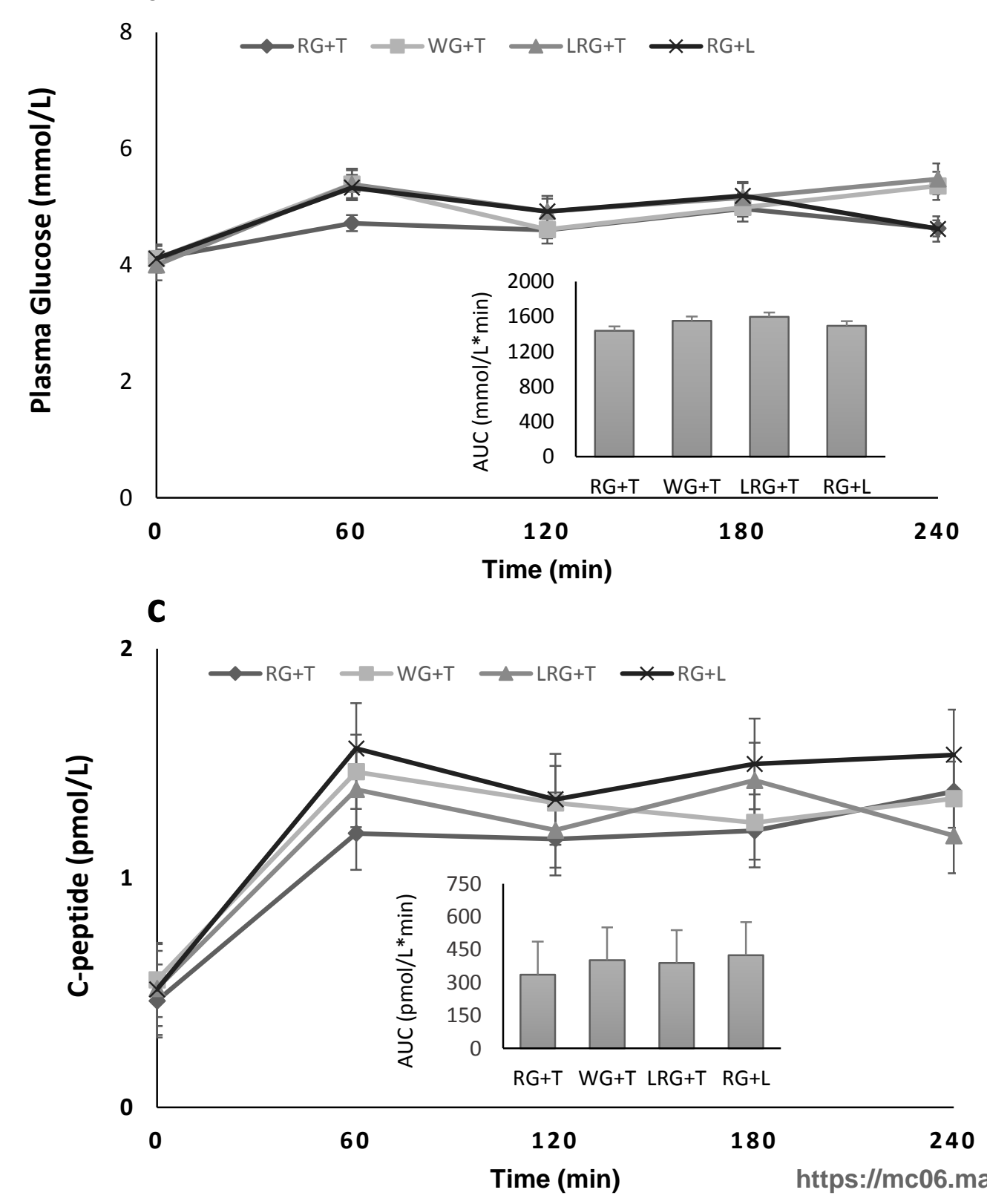

b
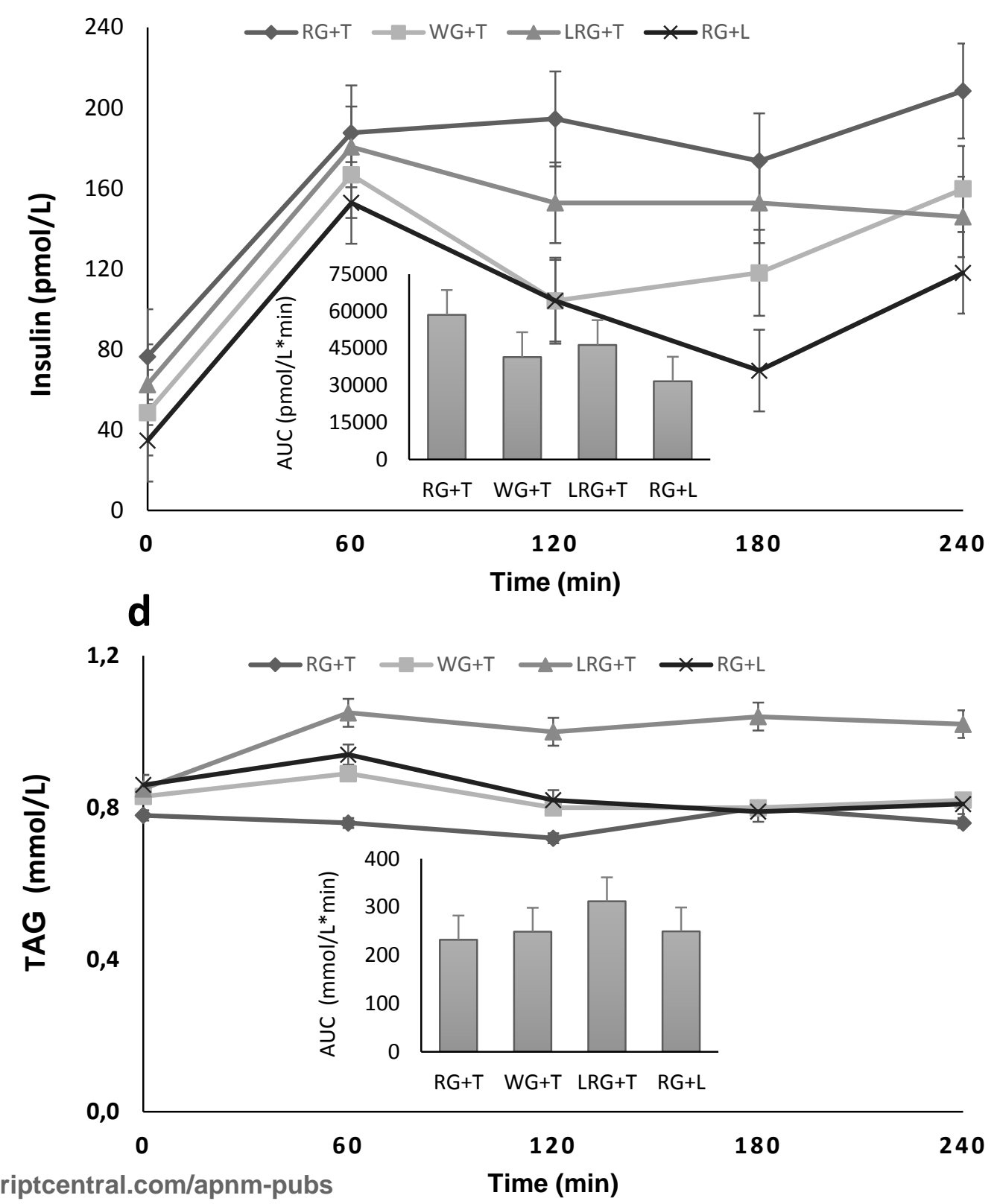